\title{
kW-class high power fiber laser enabled by active long tapered fiber
}

\author{
Chen Shi ${ }^{1,2}$, Hanwei Zhang ${ }^{1,2}$, Xiaolin Wang ${ }^{1,2}, \mathrm{Pu} \mathrm{Zhou}^{1,2}$, and Xiaojun $\mathrm{Xu}^{1,2}$ \\ ${ }^{1}$ College of Advanced Interdisciplinary Studies, National University of Defense Technology, Changsha 410073, China \\ ${ }^{2}$ Hunan Provincial Collaborative Innovation Center of High Power Fiber Laser, Changsha 410073, China \\ (Received 29 November 2017; revised 17 January 2018; accepted 27 February 2018)
}

\begin{abstract}
Compared with traditional uniform fibers, tapered fiber has numerous unique advantages, such as larger mode area, higher pump absorption, suppression to nonlinear effects, and maintaining good beam quality. In this manuscript, we have constructed an all-fiberized fiber amplifier which is based on a piece of ytterbium-doped tapered double-clad fiber (T-DCF). The fiber amplifier is operated under continuous wave (CW) regime at $1080 \mathrm{~nm}$ wavelength. The $M^{2}$ factor of the amplifier at $1.39 \mathrm{~kW}$ output power is $\sim 1.8$. The maximum output power of the system reached $1.47 \mathrm{~kW}$, which, to the best of our knowledge, is the highest output power of long tapered fiber based fiber laser system. Our result successfully verifies the potential of power scalability and all-fiberized capability of long tapered fiber, and the performance of our system can be further enhanced by fiber design optimization.
\end{abstract}

Keywords: fiber lasers and applications; laser amplifiers

\section{Introduction}

For the advantage of high slope efficiency, good beam quality, superior thermal management property and compactness, fiber lasers are widely used in the field of laser marking, material processing, medicine, communication and many other industrial applications ${ }^{[1]}$. In the past decade, output power of fiber lasers experienced a rapid boost with the development of large mode area (LMA) double-clad fibers (DCF) and cladding pump technology ${ }^{[2,3]}$. Among them, ytterbium-doped fiber lasers and amplifiers at $1 \mu \mathrm{m}$ have shown excellent optical efficiency ${ }^{[4,5]}$ and have reached over $100 \mathrm{~kW}$ output power by combining tens of $\mathrm{kW}$-level single mode fiber lasers.

Compared with traditional fiber laser oscillator, the main oscillator power amplifier (MOPA) system, which can boost the progress of fiber laser power scaling, offers an effective way to acquire high power fiber laser source with excellent beam quality by employing cascaded structure. However, further power scaling of MOPA system requires a considerable effort to suppress nonlinearities, which usually has contradiction in fiber type selection with the requirement of good beam quality. Compared to uniform LMA active fiber, T-DCF shows numerous unique advantages when

Correspondence to: X. Wang, College of Advanced Interdisciplinary Studies, National University of Defense Technology, Changsha 410073, China. Email: chinawxllin@163.com being employed as gain medium of optical amplifier, such as larger mode area, higher pump absorption, suppression to nonlinear effects, maintaining good beam quality and so on ${ }^{[6-8]}$. A number of applications using T-DCF have already been reported. T-DCF has been applied in CW fiber laser oscillator ${ }^{[9]}$, Q-switched pulsed oscillator ${ }^{[10]}, \mathrm{CW}$ fiber laser amplifier ${ }^{[1,12]}$ and ultrafast laser systems ${ }^{[13]}$. However, all of the systems in Refs. [9-13] were constructed based on the bulk optical component, which lack stability and compactness. Comparatively speaking, the all-fiberized structure has better performance in stability, compactness, thermal management and so on. Few researchers have reported their experimental results based on all-fiberized T-DCF structures. Zhou et al. has constructed an allfiberized fiber amplifier using active T-DCF ${ }^{[14]}$, and $53 \mathrm{~W}$ single frequency output at $1064 \mathrm{~nm}$ wavelength with $M^{2} \sim 1.28$ beam quality was obtained. Zhang et al. and Lou et al. have individually reported their passive T-DCF based random fiber laser system ${ }^{[15,16]}$. Up to present, the maximum output power of fiber laser based on long tapered fiber is $750 \mathrm{~W}$ with a beam quality of $M^{2} \sim 1.7^{[9]}$. A kWlevel, all-fiberized fiber laser using tapered fiber has not yet been reported.

In this manuscript, we have constructed an all-fiberized fiber amplifier, which is based on a piece of ytterbiumdoped T-DCF. The fiber amplifier was operated at $1080 \mathrm{~nm}$ wavelength under $\mathrm{CW}$ regime. The maximum output power 


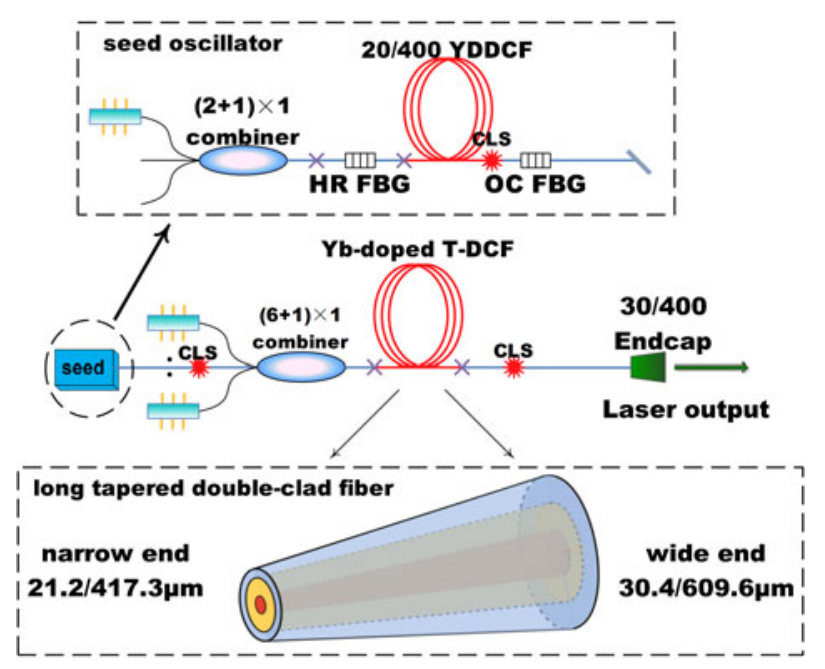

Figure 1. Schematic of the Yb-doped T-DCF based fiber amplifier.

of the system reached $1.47 \mathrm{~kW}$, which, to the best of our knowledge, is the record power of long tapered fiber based fiber laser system. The $M^{2}$ factor of the amplifier at $1.39 \mathrm{~kW}$ output power is $\sim 1.8$. Our result successfully verified the potential of power scalability and all-fiberized capability of long tapered fiber, and the performance of our system can be further enhanced by fiber design optimization.

\section{Experimental setup}

The schematic diagram of the long tapered fiber amplifier, with all-fiber connections fusion spliced, is shown in Figure 1.

As shown in Figure 1, the seed laser of the whole system is a home-made fiber laser oscillator. A $150 \mathrm{~W}$ pigtailed pumping laser diode (LD) which emits at $915 \mathrm{~nm}$ wavelength is coupled into laser cavity by a $(2+1) \times 1$ signal/pump combiner. The fiber types of signal and pump ports of the combiner are $20 / 400 \mu \mathrm{m}$ and $105 / 125 \mu \mathrm{m}$, respectively. A pair of fiber Bragg grating (FBG) whose central reflective wavelengths are $1080 \mathrm{~nm}$ are used as cavity mirrors. The reflectivity of high-reflective (HR) FBG is $99.9 \%$ while that of output-coupling (OC) FBG is $11 \%$. A piece of $\sim 39 \mathrm{~m}$ long 20/400 $\mu \mathrm{m}$ ytterbium-doped double-clad fiber (YDDCF) is inserted between grating pairs as gain medium. The oscillator can generate $\sim 100 \mathrm{~W}$ seed power and it will be directly injected into amplifier stage using a $(6+1) \times 1$ signal/pump combiner, of which the signal and pump ports are $20 / 400 \mu \mathrm{m}$ and $220 / 242 \mu \mathrm{m}$, respectively.

The gain fiber of amplifier stage is a piece of ytterbiumdoped long tapered fiber, which is $33 \mathrm{~m}$ long with core/clad diameters of $21.2 / 417.3 \mu \mathrm{m}$ and $30.4 / 609.6 \mu \mathrm{m}$ at narrow and wide end, respectively, and the core/clad NA are $0.06 / 0.46$. The narrow end of T-DCF is connected with

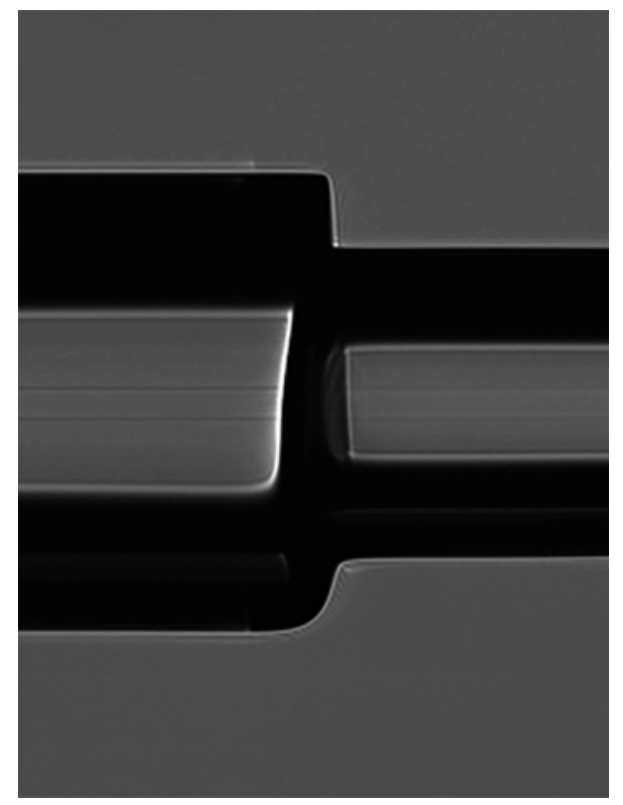

Figure 2. Splicing point of $30 / 400 \mu \mathrm{m}$ passive fiber and wide end of T-DCF.

the output port of the combiner, which means both the seed power and pump power will be injected from the narrow end. The nominal total absorption coefficient to $976 \mathrm{~nm}$ pump of the fiber is $\sim 25 \mathrm{~dB}$. The wide end of T-DCF is spliced to a piece of $30 / 400 \mu \mathrm{m}$ passive fiber, and the status of splicing point is shown in Figure 2.

In order to lead out residual pump laser, the cladding modes are stripped on the passive fiber. Finally, the passive fiber is connected to an endcap to eliminate probable harmful feedbacks at the output facet. The pump sources of the amplifier are six pigtailed LDs, each of which can generate $\sim 350 \mathrm{~W}$ pump power at $976 \mathrm{~nm}$ wavelength, and they are coupled into the amplifier through the pump ports of the combiner.

After the endcap, we put power meter, optical spectrum analyzer (OSA) and photoelectric detector (PD) to record power, optical spectrum and time domain data, respectively. The bandwidths of both PD and oscilloscope are $1 \mathrm{GHz}$ in our experiment. In the time trace measurement process, the PD with a pinhole of $1.5 \mathrm{~mm}$ diameter is placed in the center of the collimated beam to monitor the onset of transverse modal instability (TMI). From previous researches, we can know that the identification of the presence of TMI is the time domain fluctuation with frequency less than $5 \mathrm{kHz}^{[17,18]}$ and this will also be used as the criterion in our experiment.

\section{Results and discussion}

Using the experimental setup which we mentioned above, the output characteristics of the amplifier are carefully investigated. Figure 3 plots the laser power output from the 


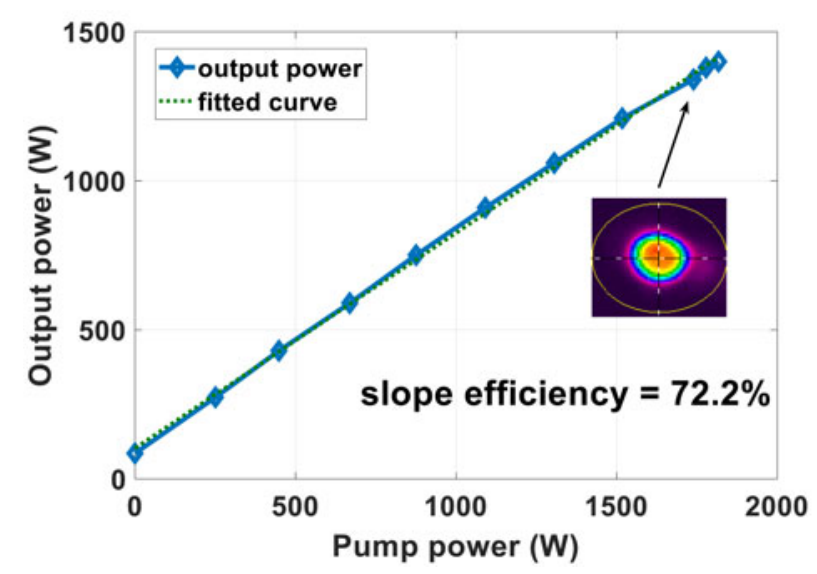

Figure 3. Measured power variation curve. Inset: measured beam pattern at $1.39 \mathrm{~kW}$ output power.

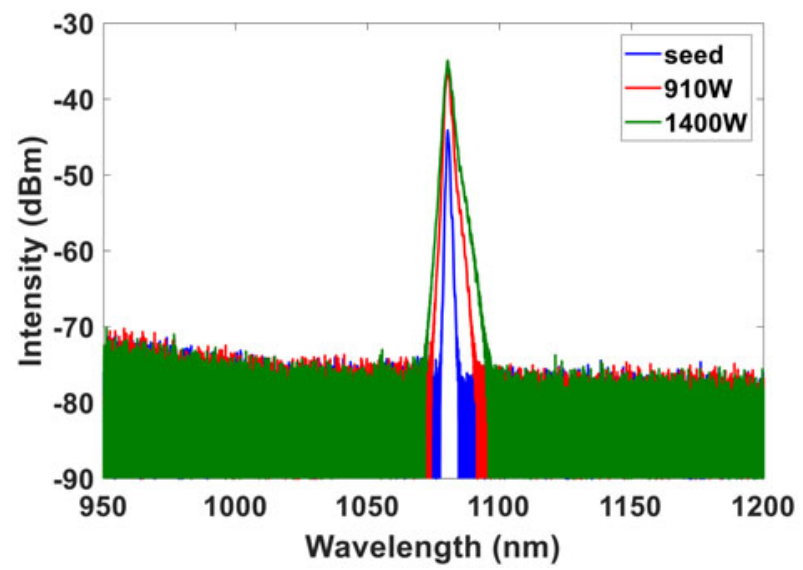

Figure 4. Measured spectrum data under different output powers of the amplifier.

endcap as a function of the pump power launched into the amplifier through the combiner.

From Figure 3, we can easily find that the output power is linearly increasing along with the variation of injected pump power. When $\sim 100 \mathrm{~W}$ seed power was launched into the fiber amplifier, $\sim 65 \mathrm{~W}$ laser power could be measured as output from the endcap. The maximum output laser power, produced by this monolithic fiber laser system, reached $\sim 1470 \mathrm{~W}$ when $\sim 1970 \mathrm{~W}$ pump power was launched into the fiber amplifier. The overall slope efficiency of the amplifier is $\sim 72.2 \%$. We have measured the output beam pattern at $1.39 \mathrm{~kW}$ output power as shown on the inset of Figure 3 and the $M^{2}$ factor is 1.8 .

The spectrum of the output laser of the amplifier is measured using an OSA with $0.05 \mathrm{~nm}$ resolution. Figure 4 shows the measured spectra of output laser under different output powers.

From Figure 4 we can find only one main peak at $1080 \mathrm{~nm}$, which is the operating wavelength of the signal laser. The spectra are perfectly clear with no pumping wavelength and nonlinear components. The $3 \mathrm{~dB}$ spectra bandwidths of seed laser, $910 \mathrm{~W}$ output and $1400 \mathrm{~W}$ output are 1.1, 1.9 and $2.3 \mathrm{~nm}$, respectively.

In order to investigate the characteristics of TMI, we also measure the time domain data of the amplifier, which is plotted in Figure 5.

Figure 5(a) shows the measured temporal traces under output power of $1.38 \mathrm{~kW}$ and $1.4 \mathrm{~kW}$. It is easy to tell that the peak-to-peak voltage of the $1.4 \mathrm{~kW}$ temporal trace is greater than that of the $1.38 \mathrm{~kW}$ temporal trace, which indicates the power interplay between fundamental mode and higher-order modes (HOMs). It is believed that this kind of power fluctuation is attributed to the occurrence of TMI. To further verify the onset of TMI, we calculate the Fourier transform of corresponding temporal traces and plot them in Figure 5(b). From the Fourier spectra, the frequency of the power fluctuation is revealed when the output power is $1.4 \mathrm{~kW}$. The notable frequency peak is around $\sim 5 \mathrm{kHz}$, which coincides with previous researches of TMI in singlepass fiber amplifiers ${ }^{[19-21]}$. From this, the occurrence of the TMI around the roll-over output power is verified and the TMI threshold in our experimental case is $1.4 \mathrm{~kW}$.

To further discuss TMI optimization of T-DCF, we have to define some parameters that we use to describe tapered fibers. We define the core radius of the narrow end and wide end of the tapered fiber as $R_{1}$ and $R_{2}$, respectively. Assume that the total length of tapered fiber is $L$, so an average tapering angle could be defined as

$$
b_{0}=\left(R_{2}-R_{1}\right) / L .
$$

Then the core radius of the fiber at a different position can be written as a function of longitudinal coordinate $z$.

$$
R(z)=\left(b_{0}-b\right) / L \cdot z^{2}+b \cdot z+R_{1} .
$$

Here, $b$ is the parabolic shaping factor that describes the shape of the taper. According to the relative value of $b$ and $b_{0}$, tapered fibers can be divided into three categories: $b>b_{0}, b=b_{0}$ and $b<b_{0}$ correspond to convex, linear and concave tapered fibers, respectively. To ensure that $R(z)$ is a monotonically increasing function, the value of $b$ has to be real and taken in the interval of $\left[0,2 b_{0}\right]$.

In the theoretical research of TMI in fiber amplifier, the growth of HOM power ratio is a key parameter in the onset judgment of TMI threshold and it could be calculated using the following equation ${ }^{[22]}$

$$
\begin{aligned}
\xi(z)= & \xi_{0} \exp \left\{\int_{z^{\prime}=0}^{z}\left[g_{1}\left(z^{\prime}\right)-g_{0}\left(z^{\prime}\right)\right] \mathrm{d} z^{\prime}\right\} \\
& +\frac{1}{4} \xi_{0} R_{N} \exp \left\{\int _ { \Omega = 0 } ^ { \Omega _ { \operatorname { m a x } } } \mathrm { d } \Omega \int _ { z ^ { \prime } = 0 } ^ { z } \left[g_{1}\left(z^{\prime}\right)-g_{0}\left(z^{\prime}\right)\right.\right. \\
& \left.\left.+\chi_{1}\left(z^{\prime}, \Omega\right) P_{0}\left(z^{\prime}\right)\right] \mathrm{d} z^{\prime}\right\}
\end{aligned}
$$



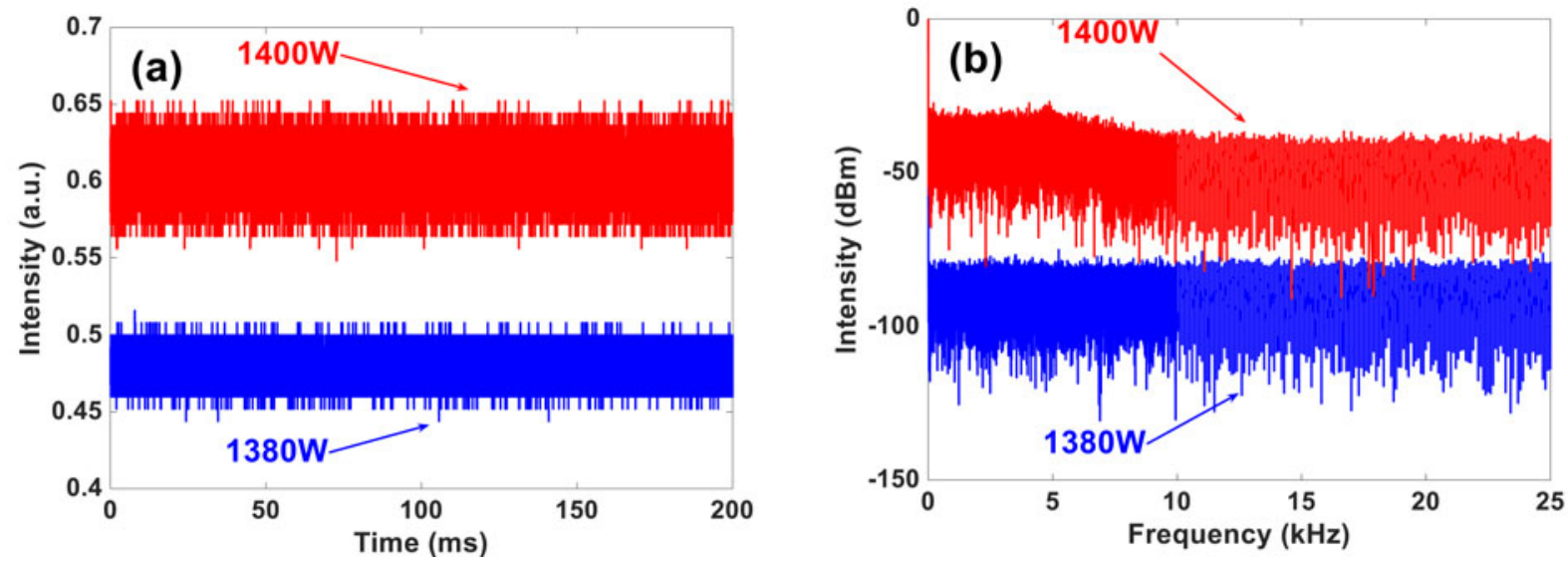

Figure 5. (a) Measured temporal trace and (b) corresponding Fourier transform of different output powers.

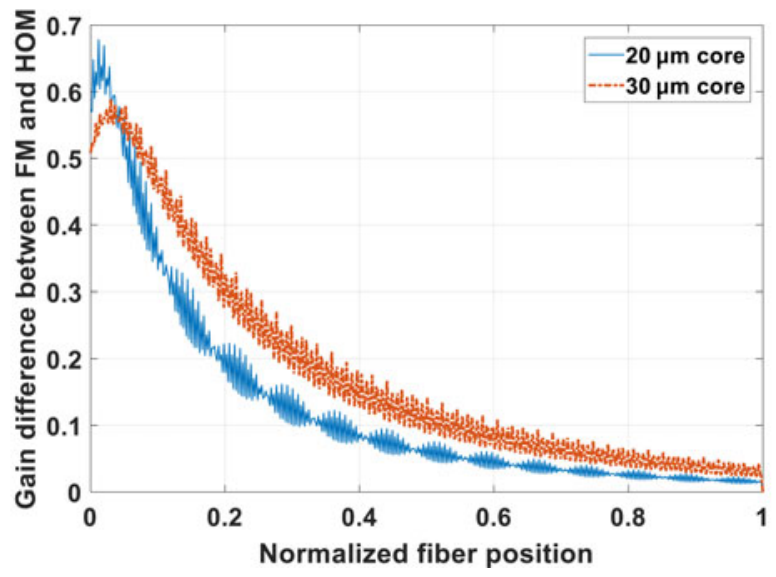

Figure 6. Calculated gain difference using co-pumping schemes of different fiber core sizes.

where $\xi_{0}$ is the HOM power ratio of seed laser; $g_{0}$ and $g_{1}$ are $\mathrm{Yb}$-gain of $\mathrm{LP}_{01}$ and $\mathrm{LP}_{11}$ modes, respectively; $R_{N}$ is the intensity noise ratio of the amplifier; $\Omega$ is the frequency shift between $\mathrm{LP}_{01}$ and $\mathrm{LP}_{11}$ modes and $\chi_{1}$ is TMI coupling coefficient. From Equation (3), we can tell a fact that the growth of HOM power ratio consists of two parts: the difference of gain between fundamental mode and HOM and nonlinear modal coupling, and both of these two parts are related to core size of gain fiber. First, as HOM will be better guided in thicker fiber, the gain difference will be greater in gain fiber with bigger core size. Figure 6 shows the simulation result of gain difference in $20 \mu \mathrm{m}$ and $30 \mu \mathrm{m}$ core size fiber.

Second, better guided HOM will cause another affection that modal distribution of HOM will be better overlapped with fiber core, which means the nonlinear coupling coefficient in thicker fiber will also be bigger than that in thinner fiber. In order to verify this conclusion theoretically, we have simulated TMI threshold variation with change of parabolic shaping factor $b$ using Equation (3). Here we choose

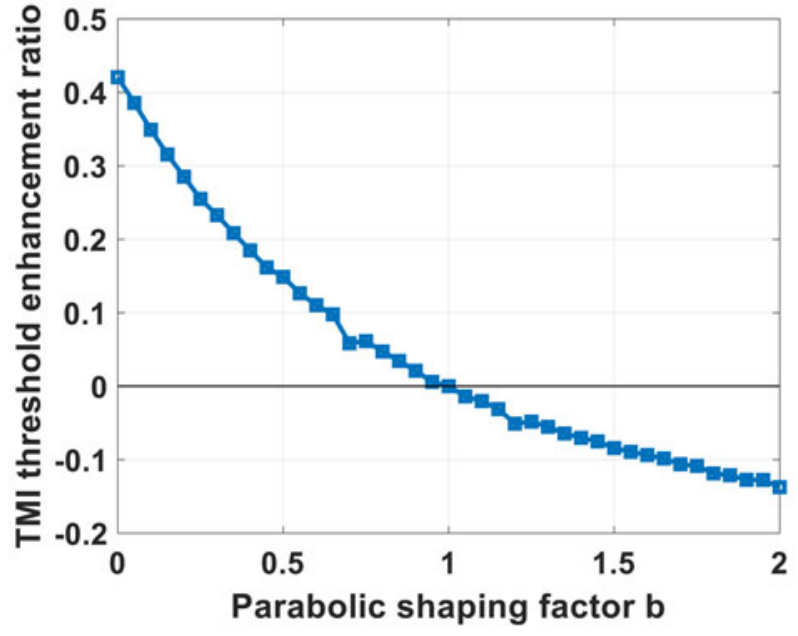

Figure 7. Relationship between parabolic shaping factor and TMI threshold.

T-DCF with $b=1$ as a standard, all the calculated thresholds will be compared with standard threshold and threshold enhancement ratio will be calculated. The simulation result is plotted in Figure 7. We can see that by using concave TDCF, the TMI threshold can be improved above $40 \%$, and the threshold of convex T-DCF is lower than linear T-DCF, which validates our theory.

From all of the above, we can conclude two possible solutions for TMI issue in T-DCF fiber laser system. First, T-DCF with smaller average core size will have a better performance in suppression of TMI. The design of the fiber we use in this case is $b \approx b_{0}$. Fabricating a piece of concave fiber $\left(b<b_{0}\right)$ which can be used in fiber amplifier with better performance in TMI is the next step of our research on TDCF. Second, we can use tandem pumping scheme in order to reduce thermal load in gain fiber. The physical origin of TMI is the heat load that generated inside the doped area of gain fiber in amplifying procedure, and quantum defect (QD) 
is the main heat source among all thermal effects. Because of that, the pumping wavelength is close to signal wavelength, and QD in tandem pumping scheme is much lower than that in LD pumping scheme. Lower QD lead to lower overall thermal load in gain fiber, so that the threshold of TMI can be effectively improved in tandem pumping configuration.

\section{Conclusion}

In summary, we have constructed an all-fiberized fiber amplifier which is based on a piece of ytterbium-doped TDCF. The seed laser of the whole system is a home-made fiber laser oscillator which can generate $\sim 100 \mathrm{~W} \mathrm{CW}$ seed laser. The fiber amplifier is operated under $\mathrm{CW}$ regime. The maximum output power of the system reached $1.47 \mathrm{~kW}$. To the best of our knowledge, it is the highest output power of long tapered fiber based fiber laser system. Our result successfully verifies the potential of power scalability and all-fiberized capability of long tapered fiber, and the performance of our system can be further enhanced by fiber design optimization.

\section{Acknowledgements}

The authors would like to thank Prof. Zhiyong Pan and CETC-23 institute for supplying T-DCF for our experiments. This work was supported by the National Natural Science Foundation of China (No. 61505260) and the National Key Research and Development Program of China (No. 2016YFB0402204).

\section{References}

1. M. N. Zervas and C. A. Codemard, IEEE J. 20, 219 (2014).

2. D. J. Richardson, J. Nilsson, and W. A. Clarkson, J. Opt. Soc. Am. B 27, B63 (2010).

3. O. G. Okhotnikov, Fiber Lasers, 3rd ed. (John Wiley \& Sons, 2012).
4. H. Yu, H. Zhang, X. Wang, J. Leng, H. Xiao, S. Guo, P. Zhou, X. Xu, and J. Chen, Appl. Opt. 54, 4556 (2015).

5. H. Xiao, J. Leng, H. Zhang, L. Huang, J. Xu, and P. Zhou, Appl. Opt. 54, 8166 (2015).

6. V. Filippov, Y. K. Chamorovskii, K. M. Golant, A. Vorotynskii, and O. G. Okhotnikov, Proc. SPIE 9728, 97280V (2016).

7. V. Filippov, Y. Chamorovskii, J. Kerttula, K. Golant, M. Pessa, and O. G. Okhotnikov, Opt. Express 16, 1929 (2008).

8. C. Shi, X. Wang, P. Zhou, X. Xu, and Q. Lu, Opt. Express 24, 19473 (2016).

9. V. Filippov, J. Kerttula, Y. Chamorovskii, K. Golant, and O. G. Okhotnikov, Opt. Express 18, 12499 (2010).

10. J. Kerttula, V. Filippov, Y. Chamorovskii, K. Golant, and O. G. Okhotnikov, Opt. Express 18, 18543 (2010).

11. J. Kerttula, V. Filippov, Y. Chamorovskii, V. Ustimchik, K. Golant, and O. G. Okhotnikov, Laser Phys. 22, 1734 (2012).

12. A. I. Trikshev, A. S. Kurkov, V. B. Tsvetkov, S. A. Filatova, J. Kertulla, V. Filippov, Y. K. Chamorovskiy, and O. G. Okhotnikov, in 2013 Conference on Lasers and Electro-Optics - International Quantum Electronics Conference (Optical Society of America, 2013), paper CJ_P_33.

13. V. Filippov, V. Ustimchik, Y. Chamorovskii, K. Golant, A. Vorotynskii, and O. G. Okhotnikov, in European Conference on Lasers and Electro-Optics (Optical Society of America, 2015), paper CJ_10_5.

14. Z. Zhou, H. Zhang, X. Wang, Z. Pan, R. Su, B. Yang, P. Zhou, and X. Xu, J. Opt. 18, 65504 (2016).

15. Z. Lou, J. Xu, L. Huang, H. Zhang, J. Leng, H. Xiao, and P. Zhou, Laser Phys. Lett. 14, 55102 (2017).

16. H. Zhang, X. Du, P. Zhou, X. Wang, and X. Xu, Opt. Express 24, 9112 (2016).

17. R. Tao, P. Ma, X. Wang, P. Zhou, and Z. Liu, Photon. Res. 3, 86 (2015).

18. H. Otto, F. Stutzki, F. Jansen, T. Eidam, C. Jauregui, J. Limpert, and A. Tünnermann, Opt. Express 20, 15710 (2012).

19. R. Tao and R. Su, Laser Phys. Lett. 14, 25101 (2017).

20. R. Tao, P. Ma, X. Wang, P. Zhou, and Z. Liu, Proc. SPIE 9255, 92550B (2015).

21. A. V. Smith and J. J. Smith, IEEE J. Quantum Electron. 20, 472 (2014)

22. I. Hu, C. Zhu, C. Zhang, A. Thomas, and A. Galvanauskas, Proc. SPIE 8601, 860109 (2013). 